\title{
On the logic of testing the independence assumption in the process-dissociation procedure
}

\author{
ELLIOT HIRSHMAN \\ University of North Carolina, Chapel Hill, North Carolina
}

\begin{abstract}
The process-dissociation procedure is designed to provide quantitative estimates of the influence of explicit and implicit memory in a variety of tasks. The procedure relies on the assumption that these two forms of memory produce independent influences on performance. Prior investigators have attempted to test this assumption by determining whether the parameter representing the influence of implicit memory (denoted $A$ ) is constant across experimental conditions. I argue that the constancy of $A$ cannot provide an appropriate test of the independence assumption, because (1) the prediction of constancy can be generated without the assumption of independence, obviating the need to posit independence; and (2) the constancy of $\boldsymbol{A}$ does not necessarily imply independence, even if one assumes that a dependency hypothesis, supplemented by ancillary assumptions (Curran \& Hintzman, 1995), predicts differences in $A$. I close by emphasizing that we can test the independence assumption by using standard procedures that compare the fit of a model that assumes independence with the fit of a model that assumes dependence.
\end{abstract}

Although initial work on implicit memory (Schacter, 1987) raised hopes of substantial theoretical and practical progress, methodological difficulties have retarded attempts to identify the information processing characteristics of implicit memory. Specifically, although there is substantial evidence that implicit memory relies on perceptual information (e.g., Roediger \& Blaxton, 1987), it has been difficult to determine whether implicit memory utilizes semantic information (e.g., Challis \& Brodbeck, 1992). A primary reason for this is that explicit memory can affect performance on tasks hypothesized to measure implicit memory. This creates an interpretive ambiguity when semantic information affects these tasks, because such effects could arise from explicit memory processes that rely on this information.

Jacoby (1991), who recognized that both explicit and implicit memory could affect most tasks, proposed the process-dissociation procedure as a quantitative means of isolating the influences of explicit and implicit memory. As discussed by Russo, Cullis, and Parkin (in press), the procedure is applied to an empirical paradigm in which subjects receive two memory tests. In one of these tests (called the inclusion test), implicit and explicit memory are hypothesized to work together to produce responses. In the other test (called the exclusion test), explicit memory is hypothesized to oppose the tendency of implicit memory

The author was supported by Research Grant SBR-9420248 from the National Science Foundation. Correspondence may be addressed to E. Hirshman, ellioth@email.unc.edu.

-Accepted by previous editor, Geoffrey R. Loftus to produce responses. The assumption that the influences of implicit and explicit memory are stochastically independent allows one to write expressions that convert performance in the inclusion and exclusion conditions into parameters representing the probability of implicit and explicit memory influencing performance. Specifically,

$$
I=R+A-(R * A)
$$

and

$$
E=A *(1-R),
$$

where $I$ represents performance in the inclusion condition, $E$ represents performance in the exclusion condition, $R$ represents the influence of explicit memory ( $R$ stands for recollection, presumably a subjective correlate of explicit memory), and $A$ represents the influence of implicit memory ( $A$ stands for automatic, presumably an information processing correlate of implicit memory).

Although this procedure has been applied widely (Jacoby, 1991; Jennings \& Jacoby, 1993; Toth, Reingold, \& Jacoby, 1994; Yonelinas, 1994), many of its basic assumptions have been severely criticized (Buchner, Erdfelder, \& Vaterrodt-Plünnecke, 1995; Curran \& Hintzman, 1995; Graf \& Komatsu, 1994; Mulligan \& Hirshman, 1997; Richardson-Klavehn, Gardiner, \& Java, 1994). One important criticism focuses on the assumption that the influences of explicit and implicit memory are stochastically independent (Curran \& Hintzman, 1995).

This assumption is both substantively strong and critical to the re-parameterization afforded by the processdissociation procedure. At the substantive level, it suggests that the probability of implicit memory's producing a response will be the same whether or not explicit mem- 
ory produces the response. This assumption is extremely strong because there are many factors (e.g., frequency) that one might expect to produce parallel influences on the probability of producing responses in implicit and explicit memory. At the level of the re-parameterization, an assumption of dependence makes it impossible to reparameterize the data from the inclusion and exclusion tests. This is because the model assuming dependence must include a parameter representing the covariation of implicit and explicit memory, forcing it to contain more parameters than there are data points.

Russo et al. (in press) have attempted to test the independence assumption by examining how a levels-ofprocessing manipulation affects estimates of $R$ and $A$. They argue, following logic proposed by Jacoby, Toth, and Yonelinas (1993) and Toth et al. (1994), that if independence holds, one would expect levels-of-processing to affect $R$, but not $A$. This is because levels-of-processing often affects performance on explicit, but not implicit, tasks (see, e.g., Graf \& Mandler, 1984), and $R$ and $A$ are supposed to measure explicit and implicit memory, respectively. Further, Russo et al. point out, following arguments proposed by Curran and Hintzman (1995), that if explicit and implicit memory are positively correlated and certain ancillary assumptions hold, one would expect spurious dissociations in which variables increase $R$, while decreasing $A$. While Toth et al. demonstrated that levels-of-processing increased $R$, but had no effect on $A$, Russo et al. demonstrated in three experiments that levelsof-processing increased $R$, but decreased $A$.

Although these results seem to pose problems for the application of the process-dissociation procedure, an alternative interpretation of these results has been proposed by Jacoby, Begg, and Toth (1997). These authors point out that subjects may be using a direct retrieval strategy that induces the paradoxical dissociation. Thus, it may still be possible to use the process-dissociation procedure, but one must use instructions that produce independent influences of explicit and implicit memory as assumed by the procedure.

While determining the validity of Jacoby et al.'s (1997) argument is important, I wish to pursue a broader point here. Specifically, I wish to emphasize that, contrary to the claims of Jacoby and his colleagues, the constancy of $A$ across experimental conditions does not necessarily support the independence assumption. Thus, prior studies in which $A$ is approximately constant (e.g., Toth et al., 1994) do not necessarily provide evidence for the independence assumption.

To reiterate the logic of prior tests, the finding that levels-of-processing (or other variables) affects $R$ but not $A$ is taken as evidence for independence. The motivation for this is that (1) $R$ and $A$ are supposed to measure explicit and implicit memory, respectively; and (2) some prior empirical studies have demonstrated that levels-ofprocessing affects tasks hypothesized to measure explicit memory, but not tasks hypothesized to measure implicit memory. This logic is problematic in two respects.

The first problem with the logic is that the prediction of constancy can be generated without the assumption of independence. This suggests that it is not necessary to posit independence in order to account for the constancy of $A$. I demonstrate how constancy of $A$ can arise without independence in two ways here. The first relies on the ancillary assumptions of Jacoby and his colleagues; the second considers the implications of a simple empirical regularity for the constancy of $A$.

First, assuming the premises proposed by Jacoby and his colleagues (see the paragraph immediately preceding the prior one), the independence assumption is not necessary to predict the constancy of $A$. Specifically, note that one still predicts the constancy of $A$ across selected variables if (1) $A$ measures implicit memory, and (2) prior work from a "pure" test demonstrates that the selected variables have no effect on implicit memory.

Second, a negative correlation between performance in the inclusion and exclusion conditions (a finding that commonly occurs in uses of the opposition paradigm) can operate to keep the value of $A$ approximately constant. A simple reason for this negative correlation is that if one is better able to identify that an item was in a specific list, increasing $I$, one might also be better able to identify that the item was from the specific list, decreasing $E$. To understand the implications of this empirical regularity for values of $A$, consider the equations for estimating $R$ and $A$ :

$$
\begin{aligned}
& R=I-E, \\
& A=E /(1-R) .
\end{aligned}
$$

Now consider what happens to the value of $A$ as $I$ changes if inclusion and exclusion performance are negatively correlated. Representing this correlation algebraically as

$$
E=1-f(I)
$$

where $f$ is any monotonically increasing function of $I$ that is bounded between 0 and 1 , gives

$$
A=[1-f(I)] /(1-R)] .
$$

Note that when $I$ (and consequently $R$ ) increases, this forces both the numerator and denominator in Equation 6 down, a factor that tends to keep the value of $A$ constant.

The preceding points suggest that the constancy of $A$ can arise from a number of factors other than independence. This obviates the need to posit independence to account for the constancy of $A$.

The second problem with the logic is that the constancy of $A$ does not necessarily imply independence, even if one assumes that a dependency hypothesis, coupled with ancillary assumptions, predicts differences in $A$ (Curran \& Hintzman, 1995). To see this, consider the following logical expression as a statement of Curran and Hintzman's view: 


$$
\begin{aligned}
& \text { (dependence and ancillary assumptions) } \\
& \rightarrow(A \text { is not constant }) \text {. }
\end{aligned}
$$

This implies:

not ( $A$ is not constant)

$\rightarrow$ not (dependence and ancillary assumptions),

which can be rewritten as

$$
\begin{aligned}
& \text { ( } A \text { is constant) } \\
& \quad \rightarrow \text { (independence) } \\
& \quad \text { or (ancillary assumptions are not true). }
\end{aligned}
$$

(Note that the or in Equation 9 represents the inclusive or.) Thus, the constancy of $A$ may indicate that certain ancillary assumptions (e.g., constancy of $A$, ironically) do not hold rather than that independence holds.

Altogether these arguments suggest that when constancy of $A$ occurs one can account for it on the basis of other assumptions, and it does not necessarily imply independence. This suggests that the constancy of $A$ is not an appropriate test of independence. However, I wish to emphasize that the preceding does not suggest that the constancy of $A$ is necessarily unimportant. Given the assumptions of Jacoby and his colleagues, $A$ 's constancy is consistent with the view that the model re-parameterization is appropriate, and this provides general support for the process-dissociation procedure (Jacoby et al., 1993).

A proponent of the process-dissociation procedure might ask, How can the data not provide evidence for the independence assumption when the process-dissociation procedure that uses this assumption is supported? To understand this, note that an appropriate re-parameterization provides evidence for the model generally, but not necessarily for any specific assumption in the model. Thus, given an appropriate data set, it is still possible that a model assuming dependence could also provide an appropriate re-parameterization, as well as a better fit.

To see this general idea in a specific historical example, note that a signal detection model that assumes that the old and new distributions have equal variance will often provide appropriate measures of sensitivity and bias (Snodgrass \& Corwin, 1988). Nonetheless, other tests (e.g., Ratcliff, Sheu, \& Gronlund, 1992) have clearly demonstrated that the assumption of unequal variance is more appropriate.

I close by emphasizing that even though the logic followed by Jacoby and his colleagues does not necessarily provide an appropriate test of the independence assumption, such a test is feasible. Since the independence model is nested within the dependence model (i.e., it sets the covariation parameter to 0 ), one can follow standard model testing procedures and examine whether there is a significant loss in fit to appropriate data when the independence assumption is imposed. This specific test of the in- dependence hypothesis provides the opportunities for fruitful model development and revision.

\section{REFERENCES}

Buchner, A., Erdfelder, E., \& Vaterrodt-Plünnecke, B. (1995). Toward unbiased measurement of conscious and unconscious memory processes within the process dissociation framework. Journal of Experimental Psychology: General, 124, 137-160.

Challis, B. H., \& BrodBeck, D. R. (1992). Levels of processing affects priming in word fragment completion. Journal of Experimental Psychology: Learning, Memory, \& Cognition, 18, 595-607.

Curran, T., \& Hintzman, D. L. (1995). Violations of the independence assumption in process dissociation. Journal of Experimental Psychology: Learning, Memory, \& Cognition, 21, 531-547.

Graf, P., \& Komatsu, S.-I. (1994). Process dissociation procedure: Handle with caution! European Journal of Cognitive Psychology, 6, 113-129.

GraF, P., \& MANDlER, G. (1984). Activation makes words more accessible, but not necessarily more retrievable. Journal of Verbal Learning \& Verbal Behavior, 23, 553-568.

JACOBY, L. L. (1991). A process dissociation framework: Separating automatic from intentional uses of memory. Journal of Memory \& Language, 30, 513-541

JACOBY, L. L., BEGG, I. M., \& ToTH, J. P. (1997). In defense of functional independence: Violations of assumptions underlying the process-dissociation procedure? Journal of Experimental Psychology: Learning, Memory, \& Cognition, 23, 485-495.

JACOBY, L. L., Toth, J. P., \& Yonelinas, A. P. (1993). Separating conscious and unconscious influences of memory: Measuring recollection. Journal of Experimental Psychology: General, 122, 139-154.

JENNINGs, J. M., \& JACOBY, L. L. (1993). Automatic versus intentional uses of memory: Aging, attention, and control. Psychology \& Aging, 8, 283-293.

Mulligan, N. W., \& Hirshman, E. (1997). Measuring the bases of recognition memory: An investigation of the process-dissociation framework. Journal of Experimental Psychology: Learning, Memory, \& Cognition, 23, 280-304.

RatCliff, R., Sheu, C.-F., \& Gronlund, S. D. (1992). Testing global memory models using ROC curves. Psychological Review, 99, 518-535.

Richardson-Klavehn, A., Gardiner, J. M., \& Java, R. I. (1994). Involuntary conscious memory and the method of opposition. Memory, 2, 1-29.

Roediger, H. L., III, \& Blaxton, T. A. (1987). Effects of varying modality, surface features, and retention interval on priming in wordfragment completion. Memory \& Cognition, 15, 379-388.

Russo, R., Cullis, A. M., \& Parkin, A. J. (in press). Consequences of violating the assumption of independence in the process dissociation procedure: A word fragment completion study. Memory \& Cognition.

SCHACTER, D. L. (1987). Implicit memory: History and current status. Journal of Experimental Psychology: Learning, Memory, \& Cognition, 13, 501-518.

SnOdGrass, J. G., \& CoRwIN, J. (1988). Pragmatics of measuring recognition memory: Applications to dementia and amnesia. Journal of Experimental Psychology: General, 117, 34-50.

Toth, J. P., Reingold, E. M., \& Jacoby, L. L. (1994). Toward a redefinition of implicit memory: Process dissociations following elaborative processing and self-generation. Journal of Experimental Psychology: Learning, Memory, \& Cognition, 20, 290-303

YoNELINAS, A. P. (1994). Receiver-operating characteristics in recognition memory: Evidence for a dual-process model. Journal of Experimental Psychology: Learning, Memory, \& Cognition, 20, 1341-1354.

(Manuscript received May 15, 1997; revision accepted for publication June $27,1997$. ) 